\title{
Limits of Stability, Tandem Walk, and Adaptation Test in Individuals with Symptomatic Knee Osteoarthritis: A Case-control Comparison with Knee OA and Healthy Knee Controls
}

\author{
Hyoungjin Park ${ }^{1, *}$, Taewoon Jung ${ }^{2}$ \\ ${ }^{1}$ Department of Kinesiology, University of Georgia, 330 River Rd 210 Movement Studies Athens, GA. 30602, USA \\ ${ }^{2}$ Department of Therapeutic Horse Riding, Jeonju Kijeon College, South Korea
}

Copyright $\bigcirc 2018$ by authors, all rights reserved. Authors agree that this article remains permanently open access under the terms of the Creative Commons Attribution License 4.0 International License

\begin{abstract}
The purpose of this study was to compare adults with knee OA and "healthy knee" controls on balance ability by matching gender, age, mass, height, and BMI. Twenty-eight adults, 14 with medically diagnosed knee osteoarthritis and 14 with "healthy knee" status, were evaluated on dynamic balance as measured by the NeuroCom Balance Master ${ }^{\circledR}$ Limits of stability, tandem walk, and adaptation test protocols. Outcomes measured reaction time, movement velocity, maximum excursion, directional control for the limits of stability and speed, step width, endpoint sway velocity for the tandem walk test, and sway energy score for the adaptation test. The results showed that the "healthy knee" control subjects exhibited statistically significantly better results on all measures tested, however, this study did suggest balance deficits in individuals with knee OA may be better assessed for fall risk if placed in the situation where body weight is put forward, as these movements better mimic their activities of daily living.
\end{abstract}

Keywords Knee Osteoarthritis, Limits of Stability, Tandem Walk, Dynamic Balance

\section{Introduction}

Osteoarthritis (OA) is the most prevalent chronic joint condition, which is characterized by a degradation of articular cartilage, sclerosis of the subchondral bone, and osteophyte formation with symptoms of joint pain and dysfunction [1]. Among US adults, this condition is most commonly noted in the knee joints [2]. People with knee OA have also shown a reduction in lower limb muscle strength, proprioception abnormality, and a significant decline of mechanoreceptors compared to individuals with "healthy knees". [1, 3, 4]. These characteristics diminish the capability of balance and the ability to initiate and correct movements which is an indicator of fall risks common among those with knee OA. As a result, adults with knee OA have reduced balance which often translates to higher fall and resulting injury rates when compared to adults without arthritis in the United States [5].

Quantifying balance impairments is an essential task for monitoring OA progression and its outcomes. Clinical balance assessments can include a variety of tests: step test, single leg stance, functional reach test, and tandem stance test provide important information on balance deficits and fall risks in individuals with OA $[6,7]$. However, these evaluation tools present with a subjective element which can make it difficult to assess the full picture of an adult's balance control [8]. Furthermore, it is also widely accepted that instrumented tests of balance allow the attainment of more accurate measurements of the participant's postural stability [9], in a more valid and reliable way (i.e. assessment of postural stability using the limits of stability (LOS) [10]. Also, abnormal balance control can be discovered by adding "safe" challenges to gait performance [11], such as having people walk in tandem to assess dynamic balance, since falls usually occur during tasks which are related to dynamic balance control $[12,13]$.

Also, systems of maintaining balance in individuals are multifactorial and are known to be affected by such things as musculoskeletal and neurological limitations, normal aging, underutilizing available sensory information, and anthropometric factors such as height, mass, and BMI [14]. Because anthropometric factors have been correlated with balance previously [15], careful consideration for this must be made by the researcher when matching experimental and control groups for studies on balance. To date, no 
comparative studies have been conducted specific to the evaluation of balance for individuals with knee OA using limits of stability and tandem walk with matching anthropometric factors. Therefore, this study aimed to compare the dynamic balance measures of individuals with knee OA using age, gender, height, mass, and BMI matched "healthy knee" control subjects.

\section{Method}

\section{Participants}

Fourteen participants (11 female, 3 male) with knee OA and 14 asymptomatic controls (11 female, 3 male) aged between 27 and 64 years were recruited through advertisements in local clubs, libraries, school listserv, and the print.

Fourteen participants in each group were calculated via an a priori power analysis using $\mathrm{G}^{*}$ Power $^{\mathrm{TM}}$ (Kiel University, Germany) to provide $80 \%$ power (1- $\beta$ ) with an effect size of approximately 1 at $\alpha=.05$ to detect statistical differences. The data used for the estimates were the movement velocity result of LOS reported in a study that was done by Clark and Rose on the dynamic balance of community-dwelling older adult fallers [16]. All participants were free from (1) a concomitant medical illness that could damage balance (e.g., neurological or significant musculoskeletal disease, Meniere's disease or other inner ear diseases, permanent lower-limb injury, or significant psychiatric disorder) and (2) the inability to carry out a tandem gait. Exclusion criteria for the knee OA group include the following: inflammatory arthritis, major lower extremity joint surgery (e.g., knee arthrotomy within the previous 6 months), any condition which severely limits local ambulation (e.g., amputation or stroke), or inability to understand and follow instructions. Participants with knee OA were matched with "healthy knee" control participants based on gender, age, height, and body mass. Exclusion criteria for the control group include the following: evidence of rheumatoid or any other type of arthritis, a history of injury to the lower extremity, or prolonged knee pain that required medication and knee surgery. Control group participants with recurring or prolonged knee pain occurring within the last month, even if pain-free on the day of testing, were excluded. Participant characteristics are presented in Table 1.

Table 1. Characteristics of OA and control participants

\begin{tabular}{|c|c|c|c|}
\hline $\begin{array}{c}\text { Participant } \\
\text { Characteristics }\end{array}$ & $\begin{array}{c}\text { Knee OA } \\
(\boldsymbol{n}=\mathbf{1 4}) \\
\text { Mean (S.D.) }\end{array}$ & $\begin{array}{c}\text { Healthy } \\
\text { Knee (n=14) } \\
\text { Mean (S.D.) }\end{array}$ & P value \\
\hline Age (yr) & $54.28( \pm 10.56)$ & $52.21( \pm 9.56)$ & 0.591 \\
\hline Height (cm) & $\begin{array}{c}165.10 \\
( \pm 10.54)\end{array}$ & $\begin{array}{c}163.65 \\
( \pm 7.83)\end{array}$ & 0.683 \\
\hline Mass $(\mathrm{kg})$ & $78.02( \pm 12.28)$ & $\begin{array}{c}75.10 \\
( \pm 11.75)\end{array}$ & 0.526 \\
\hline BMI $\left(\mathrm{kg} / \mathrm{m}^{2}\right)$ & $28.69( \pm 4.60)$ & $27.98( \pm 4.03)$ & 0.667 \\
\hline
\end{tabular}

\section{Limits of Stability (LOS)}

Limits of stability was assessed using the NeuroCom Balance Master ${ }^{\circledR}$ long plate sampling rate at $100 \mathrm{~Hz}$ (NeuroCom, Clackamas, OR). The limits of stability (LOS) test utilizes a long forceplate (18" x 60") with force transducers connected to a computer monitor for visual performance. During testing, each participant shifted his/her weight to move a cursor projected on the computer screen to one of eight targets surrounding a center starting position: 1) forward, 2) forward-right; 3) right, 4) right-backward; 5) backward, 6) left-backward; 7) left, and 8) forward-left. The participant was instructed to move the cursor to each appropriate target as quickly, accurately, and as far as they could in 8 seconds. Based on the eight trials of the LOS test, four parameters were calculated: reaction time (RT), which is the time from the signal movement to the first participant's movement (seconds), movement velocity (MV), which is the average speed of the center of gravity (COG) movement (degrees per second), maximum excursion (MXE), or the farthest displacement toward the designed target reached by the COG during the test measured in displacement percentage, and directional control (DC), which was expressed as a percentage as well. There was a comparison between the amount of movement in the intended direction (toward the target) and the amount of extraneous movement (away from the target). RT, MV, MXE, and DC were analyzed by four directions (forward, backward, right, and left).

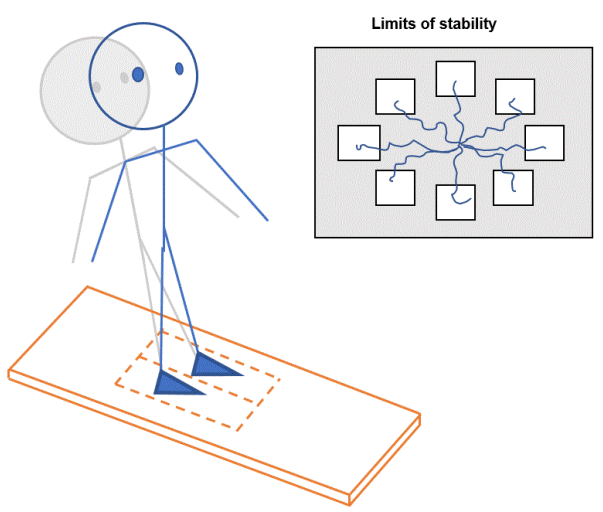

Figure 1. Limits of Stability

\section{Tandem walk test}

Participants were given a verbal description of the test followed by a visual demonstration, as well as a practice trial, before performing the test. The tandem walk test had participants walk heel to toe from one end of the forceplate to the other. Three trials were performed with a 10 -second rest between trials. Three parameters were used for the tandem walk test: speed $(\mathrm{cm} / \mathrm{s})$, which was the velocity in centimeters per second of forward progression, step width $(\mathrm{cm})$, which was the lateral distance in centimeters between the left and right feet on successive steps, and endpoint sway velocity $(\% / s)$, which was the velocity in degrees per second of the anterior/posterior component of COG sway 
for 5 seconds beginning when the patient terminates walking.

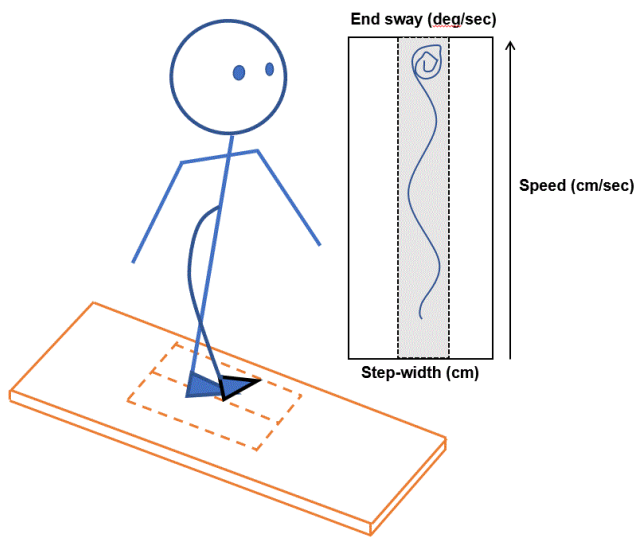

Figure 2. Tandem walk

\section{Adaptation test (ADT)}

The adaptation test (ADT) was administered using the NeuroCom EquiTest ${ }^{\circledR}$ (NeuroCom International, Clackamas, OR) to obtain the center of pressure data needed to calculate measures of balance. The software (Version 8.5 NeuroCom, A Division of Natus, Clackmas, Oregon, USA) controls the fore-aft tilt of the two forceplates (each 9" x 18"). The electrical signals from the forceplates were collected at a sampling rate of $100 \mathrm{~Hz}$ $(A D C=12$ bit $)$. Signals were filtered using a $2^{\text {nd }}$ order Butterworth filter (cutoff frequency $=0.85 \mathrm{~Hz}$ ). The ADT consisted of two different conditions (toes-up and toes-down) with five trials of each condition, and it assessed the participants' ability to minimize sway when exposed to surface irregularities and unexpected changes in support surface declination [18]. Sequences of platform rotations in the toes-up or toes-down direction produced automatic motor responses. For each platform rotation trial, a sway energy score (SES), which assesses how well the participants minimized anterior-posterior sway, quantified the magnitude of the force response required to overcome induced postural instability. The initial disruptive responses were corrected by secondary responses in the opposing muscles during the first (unexpected) trials. With each subsequent trial, initial responses were attenuated, and secondary responses were strengthened to reduce overall sway. The last of the five trials was used for analysis. Also, it is assumed that as each trial progresses, the participants learned what to expect and the energy score would improve. A smaller sway energy score indicated the ability to react more efficiently.

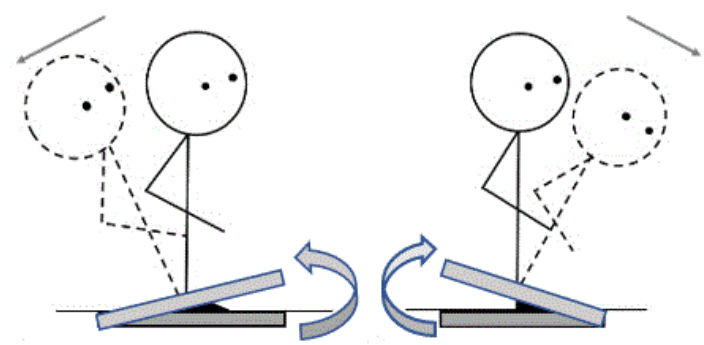

Figure 3. Toes-up and Toes-down rotations

\section{Statistical analysis}

Demographic variables assessed for this study included (a) gender, (b) age, (c) height, (d) mass, and (e) body mass index (BMI). These data, as well as the limits of the stability test and tandem walk test results, were analyzed using the t-Student test. The results of the evaluation of the significance of the differences were represented by the significance coefficient $(p)$. The level of statistical significance was indicated by: “*” $-p<0.05$.

\section{Ethical aspects}

The study was conducted according to the Declaration of Helsinki, and informed/written consent was obtained from all participants. The research protocol was approved by the university's Institutional Review Board.

\section{Results}

Independent $t$-tests were conducted to compare the dynamic balance in individuals with knee OA and healthy knee controls. The reaction time for the limits of stability is shown in Table 2. It was noticed that the reaction time was significantly different on knee OA for the forward direction $(p=0.027)$. However, there was no significant effect of knee OA in backward, right, and left directions. This parameter was faster for the healthy knee group. Also, movement velocity was statistically faster for the healthy knee group than the knee OA group in only the forward direction $(p=0.043)$.

Table 2. Reaction time (LOS) Unit: sec.

\begin{tabular}{|c|c|c|c|c|c|c|}
\hline \multirow{2}{*}{ Direction } & \multicolumn{2}{|c|}{ Knee OA } & \multicolumn{2}{c|}{ Healthy Knee } & \multirow{2}{*}{$\boldsymbol{t}$ P value } \\
\cline { 2 - 7 } & Mean & SD & Mean & SD & & \multirow{2}{*}{$0.016^{*}$} \\
\hline Forward & 1.02 & 0.24 & 0.80 & 0.22 & 2.589 & 0.274 \\
\hline Backward & 0.81 & 0.31 & 0.68 & 0.28 & 1.118 & 0.411 \\
\hline Right & 0.95 & 0.32 & 0.84 & 0.37 & 0.836 & 0.210 \\
\hline Left & 0.91 & 0.38 & 0.74 & 0.32 & 1.28 & 0 \\
\hline
\end{tabular}

* Statistically significant mean difference $(p<0.05)$, lower scores represent better performance 
Table 3. Movement velocity (LOS) Unit: Unit: $\% / \mathrm{sec}$

\begin{tabular}{|c|c|c|c|c|c|c|}
\hline \multirow{2}{*}{ Direction } & \multicolumn{2}{|c|}{ Knee OA } & \multicolumn{2}{c|}{ Healthy Knee } & \multirow{2}{*}{$\boldsymbol{t}$} & \multirow{2}{*}{$\boldsymbol{P}$ value } \\
\cline { 2 - 6 } & Mean & SD & Mean & SD & & \multirow{2}{*}{$0.046^{*}$} \\
\hline Forward & 3.41 & 1.24 & 4.62 & 1.76 & -2.102 & 0.375 \\
\hline Backward & 2.83 & 1.08 & 3.28 & 1.52 & -0.903 & 0.835 \\
\hline Right & 5.42 & 2.09 & 5.60 & 2.39 & -0.210 & 1.000 \\
\hline Left & 5.83 & 2.63 & 5.83 & 2.17 & 0.000 & \\
\hline
\end{tabular}

* Statistically significant mean difference $(p<0.05)$, higher scores represent better performance

Table 4 shows the MXE and DC results for both groups. The LOS test showed significant differences in MXE ( $\mathrm{p}=0.035)$ and DC ( $p=0.026$ ) values in the forward direction between groups. However, there was no significant effect of knee OA in backward, right, and left directions. This parameter was higher for the healthy knee group. Also, the MXE was statistically higher for the knee OA group than the healthy knee group in only the right direction $(\mathrm{p}=0.025)$.

Table 4. Maximum excursion (MXE) and Directional control (DC) Unit: \%

\begin{tabular}{|c|c|c|c|c|c|c|c|}
\hline \multirow{2}{*}{ Parameter } & \multirow{2}{*}{ Direction } & \multicolumn{2}{|c|}{ Knee OA } & \multicolumn{2}{|c|}{ Healthy Knee } & \multirow{2}{*}{$t$} & \multirow{2}{*}{$P$ value } \\
\hline & & Mean & SD & Mean & SD & & \\
\hline \multirow{4}{*}{ MXE } & Forward & 73.14 & 15.52 & 86.71 & 16.66 & -2.230 & $0.035^{*}$ \\
\hline & Backward & 63.71 & 18.14 & 64.64 & 25.58 & -0.111 & 0.913 \\
\hline & Right & 107.71 & 16.94 & 94.78 & 11.33 & 2.374 & $0.025^{*}$ \\
\hline & Left & 101.78 & 19.43 & 99.64 & 16.04 & 0.318 & 0.753 \\
\hline \multirow{4}{*}{$\mathrm{DC}$} & Forward & 69.36 & 13.53 & 78.93 & 6.88 & -2.359 & $0.026^{*}$ \\
\hline & Backward & 57.71 & 16.77 & 48.36 & 19.82 & 1.349 & 0.189 \\
\hline & Right & 72.50 & 8.03 & 71.86 & 7.09 & 0.224 & 0.824 \\
\hline & Left & 69.78 & 13.36 & 68.57 & 12.04 & 0.253 & 0.803 \\
\hline
\end{tabular}

* Statistically significant mean difference $(p<0.05)$, higher scores represent better performance

The tandem walk (TM) parametric values are shown in Table 5. It was noticed that the tandem walk tests were statistically significantly different in terms of knee OA for step-width $(p=0.045)$ and speed $(p=0.008)$. However, there was no statistically significant effect of knee OA in end-sway.

Table 5. Tandem walk

\begin{tabular}{|c|c|c|c|c|c|c|}
\hline \multirow{2}{*}{ Parameter } & \multicolumn{2}{|c|}{ Knee OA } & \multicolumn{2}{c|}{ Healthy Knee } & \multirow{2}{*}{$\boldsymbol{t}$} & \multirow{2}{*}{$\boldsymbol{P}$ value } \\
\cline { 2 - 6 } & Mean & SD & Mean & SD & & \\
\hline Step-width $^{\mathrm{a}}(\mathrm{cm})$ & 10.30 & 5.02 & 7.26 & 1.37 & 2.189 & $0.045^{*}$ \\
\hline Speed $^{\mathrm{b}}(\mathrm{cm} / \mathrm{sec})$ & 21.76 & 9.66 & 31.86 & 8.94 & -2.872 & $0.008^{*}$ \\
\hline End-sway $^{\mathrm{a}}(\% \mathrm{sec})$ & 5.26 & 3.85 & 4.26 & 1.34 & 0.912 & 0.370 \\
\hline
\end{tabular}

* Statistically significant mean difference $(p<0.05)$

${ }^{a}$ lower scores represent better performance, ${ }^{b}$ higher scores represent better performance

The results of the adaptation test were shown in Table 6. The individuals with knee OA had a significantly higher SES than the healthy knee controls in Toes-down $(p=0.031)$.

Table 6. Sway energy score (adaptation test) Unit: SES.

\begin{tabular}{|c|c|c|c|c|c|c|}
\hline & \multicolumn{2}{|c|}{ Knee OA } & \multicolumn{2}{|c|}{ Healthy Knee } & \multirow{2}{*}{$t$} & \multirow{2}{*}{$P$ value } \\
\hline & Mean & SD & Mean & SD & & \\
\hline Toes-up $\left(5^{\text {th }}\right)$ & 65.21 & 12.40 & 60.14 & 14.13 & 1.009 & 0.322 \\
\hline Toes-down $\left(5^{\text {th }}\right)$ & 54.78 & 19.01 & 41.57 & 10.54 & 2.274 & $0.031^{*}$ \\
\hline
\end{tabular}

* Statistically significant mean difference $(\mathrm{p}<$ or $=0.05)$, lower scores represent better performance 


\section{Discussion}

The purpose of the current study was to compare the dynamic balance between people with knee OA and matched "healthy knee" controls. The study showed statistically significant differences between knee OA and control groups in reaction time, movement velocity, maximum excursion, directional control (forward direction), step-width, tandem walk speed, and sway energy score.

With respect to the limits of stability, it quantifies movement characteristics associated with the ability the participants have to voluntarily change spatial position and to maintain stability at that position [18]. Instability during activities of daily living, such as walking and reaching for objects, may be the result of the inability to voluntarily shift the body toward limits of stability [9]. Individuals with knee OA had impaired postural control primarily while shifting their body weight toward the forward direction confirmed by significantly diminished scores in RT, MV, MXE, and DCL compared to healthy knee controls in the LOS test. As these findings suggest, individuals with knee OA had impaired postural control on the forward direction. Vulnerability in this manner is intensified by the observation of a longer reaction time, slower movement velocity, and decreased movement excursion toward the target in the forward direction. The average reaction time in the forward direction of the knee OA group was reduced by $21.57 \%$, and the average movement velocity was reduced by $35.48 \%$ as compared to "healthy knee" controls. In addition, sway energy scores were higher, which implies that the knee OA group displayed the ability to react less efficiently. Arvidsson, Eriksson, Knutsson, and Arner [19] concluded that pain might reflexively inhibit the activation of voluntary muscles around the knee; as a result, efficient and timely motor responses for maintaining balance could be compromised.

Further, during the automatic response, joint pain changes the responses and affects the muscle activity [20]. Increased time to return to an equilibrium position was the result of experimentally-induced thigh pain [21]. The literature reported that the individuals with knee OA showed prolonged reaction time [22]. In this study, the outcome of the analysis on the reaction time between individuals with knee OA and controls was in agreement with previous research, such as that conducted by Hofmann et al. [23]. At the same time, the reduced movement velocity recorded in the knee OA group in the current study could be an indication that these individuals are more cautious, resulting in slow movement while performing activities that can potentially compromise their balance. This result suggests that prior exposure to knee pain in individuals with knee OA equates to slower performance of the entire movement or a task. It appeared that individuals with knee OA experienced greater challenges performing the task in the forward direction. At the same time, the performance of the individuals with knee OA in the other directions did not differ from healthy knee controls that may be explained by pain and muscular weakness, which are the main characteristics of people with knee OA. All knee OA participants had mild to moderate knee joint pain when they were assessed. Additionally, it appears that the individuals with knee $\mathrm{OA}$ had some pain during the execution of body movements in a forward direction. Joint pain associated with knee OA might play a role in a reduced ability to maintain balance. Also, individuals with knee OA have shown that they have reduced levels of muscular leg strength, especially quadriceps, hamstring, and hip muscle strength [24]. Functionally, the weakness of muscular leg strength limits performance and independence in movements that require body support and position changes, which may also precipitate the risk of falls [25]. However, additional research is necessary to further examine this issue. These findings, therefore, suggest that it is necessary to pay close attention to restoring the ability of individuals with knee OA to lean in the forward direction during balance rehabilitation.

As several studies have demonstrated the importance of lateral stability in relation to risk of falling, the observed difference in the tandem walk test is a notable finding. Older people who fall have been shown to exhibit increased lateral sway when standing in bipedal [26], near-tandem [27], and unipedal positions [28], as well as an increased stride width when walking [29]. The current study showed that step-width when performing the tandem walk was significantly narrower in healthy knee controls, indicating that healthy knee controls had less of a need for more lateral foot placement to control the lateral displacement of the center of mass. Also, the Adaptation Test provides measurements used to describe a participant's ability to stabilize his or her balance using a minimum force when he or she successively shifted off balance. The mean ADT Toes Down $5^{\text {th }}$ subscore of the knee OA group population was significantly geater than that of a healthy knee group, suggesting that when successively shifting forward and off balance, the individuals with knee OA used significantly greater force to maintain balance in comparison to healthy knee controls. In conclusion, balance deficits can be identified in individuals with knee OA using LOS and a tandem walk test. This observation should be taken into account in fall prevention, and participants especially should be placed in a situation where body weight is put forward, as these are found in daily life. The findings of this study have clinical implications that are significant in terms of the understanding and management of individuals with knee $\mathrm{OA}$ and can contribute to the current body of research by complementing the extant data. Also, this study had some limitations. First, the criteria for diagnosis of knee OA might be different depending on each knee OA person's physician since the diagnosis of knee OA was based on 
several factors, including the individual's age, history, and symptoms. It may have caused individuals with asymptomatic osteoarthritis to be placed in the control group. Second, due to the various tasks, the author did not want to risk fatigue and, therefore, used one trial with practice trials rather than several trials for balance tests. This may have caused measurement errors in the data, however, the magnitude of the difference on balance was larger than the measurement error, indicating this was a true difference.

\section{REFERENCES}

[1] van der Pas, S., Castell, M. V., Cooper, C., Denkinger, M., Dennison, E. M., Edwards, M. H., Deeg, D. J. (2013). European project on osteoarthritis: design of a six-cohort study on the personal and societal burden of osteoarthritis in an older European population. Bmc Musculoskeletal Disorders, 14, 138. doi: 10.1186/1471-2474-14-138

[2] Wylde, V., Palmer, S., Learmonth, I. D., \& Dieppe, P. (2012). Somatosensory abnormalities in knee OA. Rheumatology (Oxford), 51(3), 535-543. doi: 10.1093/rheu matology/ker343

[3] Roos, E. M., Herzog, W., Block, J. A., \& Bennell, K. L. (2011). Muscle weakness, afferent sensory dysfunction and exercise in knee osteoarthritis. Nature reviews. Rheumatology, 7(1), 57-63.

[4] Tarigan, T. J. E., Kasjmir, Y. I., Atmakusuma, D., Lydia, A., Bashiruddin, J., Kusumawijaya, K., \& Prihartono, J. (2009). The degree of radiographic abnormalities and postural instability in patients with knee osteoarthritis. Acta medica Indonesiana, 41(1), 15-19.

[5] Barbour, K. E., Stevens, J. A., Helmick, C. G., Luo, Y.-H., Murphy, L. B., Hootman, J. M., Sugerman, D. E. (2014). Falls and fall injuries among adults with arthritis - United States, 2012. MMWR: Morbidity \& Mortality Weekly Report, 63(17), 379-383.

[6] Hatfield, G., Hammond, C., \& Hunt, M. A. (2015). Clinical tests of balance in the knee osteoarthritis population: A systematic review. Osteoarthritis and Cartilage, 23, A374. doi: 10.1016/j.joca.2015.02.688

[7] Khalaj, N., Abu Osman, N. A., Mokhtar, A. H., Mehdikhani, M., \& Wan Abas, W. A. (2014). Balance and risk of fall in individuals with bilateral mild and moderate knee osteoarthritis. Plos One, 9(3), e92270. doi: 10.1371/journal.pone. 0092270

[8] Kim, H.-S., Yun, D. H., Yoo, S. D., Kim, D. H., Jeong, Y. S., Yun, J.-S., Choi, S. H. (2011). Balance control and knee osteoarthritis severity. Annals of Rehabilitation Medicine, 35(5), 701-709. doi: 10.5535/arm.2011.35.5.701

[9] Ganesan, M., Kanekar, N., \& Aruin, A. S. (2015). Direction-specific impairments of limits of stability in individuals with multiple sclerosis. Annals of Physical and Rehabilitation Medicine, 58(3), 145-150. doi: https://doi.or $\mathrm{g} / 10.1016 /$ j.rehab.2015.04.002
[10] Pickerill, M. L., \& Harter, R. A. (2011). Validity and reliability of limits-of-stability testing: a comparison of 2 postural stability evaluation devices. J Athl Train, 46(6), 600-606.

[11] Salzman, B. (2010). Gait and balance disorders in older adults. American Family Physician, 82(1), 61-68.

[12] Chu, L. W., Chi, I., \& Chiu, A. Y. (2005). Incidence and predictors of falls in the Chinese elderly. Annals of the Academy of Medicine, Singapore, 34(1), 60-72.

[13] Hinman, R. S., Bennell, K. L., Metcalf, B. R., \& Crossley, K. M. (2002). Balance impairments in individuals with symptomatic knee osteoarthritis: a comparison with matched controls using clinical tests. Rheumatology (Oxford), 41(12), 1388-1394.

[14] Alonso, A. C., Brech, G. C., Bourquin, A. M., \& Greve, J. M. (2011). The influence of lower-limb dominance on postural balance. Sao Paulo Medical Journal, 129(6), 410-413.

[15] Greve, J. M. D., Cug, M., Dulgeroglu, D., Brech, G. C., \& Alonso, A. C. (2013). Relationship between anthropometric factors, gender, and balance under unstable conditions in young adults.

[16] Clark, S., \& Rose, D. J. (2001). Evaluation of dynamic balance among community-dwelling older adult fallers: A generalizability study of the limits of stability test. Archives of Physical Medicine and Rehabilitation, 82(4), 468-474. doi: https://doi.org/10.1053/apmr.2001.21859

[17] NeuroCom, N. M. I. (2008). Balance Manager Systems; Clinical Interpretation Guide.

[18] Faraldo-García, A., Santos-Pérez, S., Rossi-Izquierdo, M., Lirola-Delgado, A., Vaamonde-Sánchez-Andrade, I., del-Río-Valeiras, M., \& Soto-Varela, A. (2016). Posturographic limits of stability can predict the increased risk of falls in elderly patients with instability? Acta Oto-Laryngologica, 136(11), 1125-1129. doi: 10.1080/00016489.2016.1201591

[19] Arvidsson, I., Eriksson, E., Knutsson, E., \& Arner, S. (1986). Reduction of pain inhibition on voluntary muscle activation by epidural analgesia. Orthopedics, 9(10), 1415-1419.

[20] Takacs, J., Carpenter, M. G., Garland, S. J., \& Hunt, M. A. (2013). The role of neuromuscular changes in aging and knee osteoarthritis on dynamic postural control. Aging and Disease, 4(2), 84-99.

[21] Hirata, R. P., Ervilha, U. F., Arendt-Nielsen, L., \& Graven-Nielsen, T. (2011). Experimental muscle pain challenges the postural stability during quiet stance and unexpected posture perturbation. The Journal of Pain: Official Journal of the American Pain Society, 12(8), 911-919. doi: 10.1016/j.jpain.2011.02.356

[22] von Bernstorff, M., Feierabend, M., Jordan, M., Glatzel, C., Ipach, I., \& Hofmann, U. K. (2017). Radiographic hip or knee osteoarthritis and the ability to drive. Orthopedics, 40(1), e82-e89. doi: 10.3928/01477447-20160915-05

[23] Hofmann, U. K., Jordan, M., Rondak, I., Wolf, P., Kluba, T., \& Ipach, I. (2014). Osteoarthritis of the knee or hip significantly impairs driving ability (cross-sectional survey). Bmc Musculoskeletal Disorders, 15, 20-20. doi: 10.1186/1471-2474-15-20 
[24] Alnahdi, A. H., Zeni, J. A., \& Snyder-Mackler, L. (2012). Muscle impairments in patients with knee osteoarthritis. Sports Health, 4(4), 284-292. doi: 10.1177/1941738112445 726

[25] Carter, N. D., Khan, K. M., Mallinson, A., Janssen, P. A., Heinonen, A., Petit, M. A., \& McKay, H. A. (2002). Knee extension strength is a significant determinant of static and dynamic balance as well as quality of life in older community-dwelling women with osteoporosis. Gerontology, 48(6), 360-368. doi: 10.1159/000065504

[26] Maki, B. E., Holliday, P. J., \& Topper, A. K. (1994). A prospective study of postural balance and risk of falling in an ambulatory and independent elderly population. Journals of
Gerontology, 49(2), M72-84.

[27] Lord, S. R., Rogers, M. W., Howland, A., \& Fitzpatrick, R. (1999). Lateral stability, sensorimotor function and falls in older people. Journal of the American Geriatrics Society, 47(9), 1077-1081.

[28] Crosbie, W. J., Nimmo, M. A., Banks, M. A., Brownlee, M. G., \& Meldrum, F. (1989). Standing balance responses in two populations of elderly women: A pilot study. Arch Phys Med Rehabil, 70(10), 751-754.

[29] Maki, B. E. (1997). Gait changes in older adults: predictors of falls or indicators of fear. Journal of the American Geriatrics Society, 45(3), 313-320. 